\title{
Flow rate-controlled pipetting for microfluidics: second-generation flexible hydraulic reservoir (FHRv2)
}

\author{
Atakan Atay ${ }^{1,2} \cdot$ Alper Topuz $^{1} \cdot$ Büşra Sarıarslan ${ }^{1,2} \cdot$ Ender Yıldırım $^{3} \cdot$ Jérôme Charmet $^{4} \cdot$ Kevin Couling $^{4}$. \\ Barbaros Çetin ${ }^{1,2}$ (D)
}

Received: 27 August 2020 / Accepted: 10 November 2020 / Published online: 4 January 2021

○) Springer-Verlag GmbH Germany, part of Springer Nature 2021

\begin{abstract}
A critical component of microfluidic technology is the fluid pumping mechanism. Syringe and pressure pumps are typically used in the lab environment; however, their operations generate considerable dead volume that is often larger than the volume of the chip itself, leading to considerable waste of precious sample. As an alternative, pipetting allows for precise liquid dispensing with zero dead volume; however, it has a limited flow control. Recently, we have introduced a low-cost sample loading interface with zero dead-volume named flexible hydraulic reservoir (FHR). In this study, we present a secondgeneration FHRv2 that combines continuous pumping, zero-dead volume and the versatility of pipetting. The performance of FHRv 2 is tested against a syringe pump at flow rates ranging between 20 and $60 \mu \mathrm{L} / \mathrm{min}$. It demonstrated smoother operation and identical transient time to reach steady flow rate as confirmed by a mathematical model developed for the occasion. Importantly, we also demonstrate that the FHRv2 prevents sedimentation-induced artifacts typically encountered in typical syringe pumps when dispensing particles. Finally, we demonstrate the fabrication of the FHRv2 concept with injection molding using a 3D-printed mold. Overall, our FHRv2 offers a low-cost and versatile solution for zero-volume liquid handling in microfluidic devices.
\end{abstract}

Keywords Zero dead volume $\cdot$ Precise liquid dispensing $\cdot$ Flow-rate controlled pipetting

\section{Introduction}

Electronic supplementary material The online version of this article (https://doi.org/10.1007/s10404-020-02402-x) contains supplementary material, which is available to authorized users.

Barbaros Çetin

barbaros.cetin@bilkent.edu.tr

1 Microfluidic and Lab-on-a-chip Research Group, Mechanical Engineering Department, Bilkent University, 06800 Ankara, Turkey

2 UNAM-National Nanotechnology Research Center and Institute of Materials Science and Nanotechnology, Bilkent University, 06800 Ankara, Turkey

3 Micromanufacturing Laboratory, Mechanical Engineering Department, Middle East Technical University, 06800 Ankara, Turkey

4 Warwick Manufacturing Group (WMG), University of Warwick, Coventry CV4 7AL, UK
Microfluidics has enabled advances in numerous applications including synthesis and analysis of chemicals and biological materials, point-of-care (POC) testing (Chin et al. 2012), drug delivery, (Nisar et al. 2008; Herrlich et al. 2012) and cell handling (Moorthy and Beebe 2003; Baraket et al. 2013) as well as organs-on-a-chip platforms that (Bhatia and Ingber 2014; Huh et al. 2013) mimic in vivo environments. Although passive techniques such as capillary pumping (Zimmermann et al. 2007) or gravity (Albrecht and Bargmann 2011) may sometimes be employed for driving the liquid, long-term, stable delivery of fluids into the microfluidic channel network typically requires a pumping system. In laboratory environment, the most commonly used sources of pumping mechanisms are syringe and pressure pumps. However, these pumps that reply on tubing to connect the microfluidic chips, typically introduce considerable dead volume during operation. To overcome the dependence on bulky external hardware and to minimize the dead volume, a number of micro-pumps have been proposed. The 
common desired characteristics of these pumping systems include (1) flow control, (2) a wide range of flow rates, (3) an appropriate back pressure and ideally (4) low cost. Some micro-pumps employ moving mechanical parts, typically an oscillating diaphragm actuated by a number of mechanisms, including hand power (Gong et al. 2012; Weibel et al. 2007), electrostatic (Ni et al. 2014), electromagnetic (Amrani et al. 2018; Rusli et al. 2018; Mi et al. 2020; Tang et al. 2020), piezoelectric (Aggarwal et al. 2016; Ye et al. 2018; Ma et al. 2019), thermo-pneumatic (Kim et al. 2005) forces or rotary motion of vanes and gears (Sen et al. 1996). Even though diaphragms made of soft material are utilized most of the time, (Ni et al. 2014; Amrani et al. 2018; Rusli et al. 2018; Mi et al. 2020; Tang et al. 2020; Chen and Santiago 2002; Aggarwal et al. 2016; Ye et al. 2018; Ma et al. 2019), thermoplastic elastomers were also implemented, which enabled production of the device by common mass production techniques (Zhou et al. 2010; Guevara-Pantoja et al. 2018). The micro-pumps employing moving mechanical parts require either miniature valves with moving parts or non-moving nozzle/diffusers to rectify the fluid flow. Owing to the oscillating or reciprocating nature of the actuation, a pulsation (i.e. back flow) is present to some extent for these types of pumps. Although valveless design is simpler to fabricate, pulsating flow is even more pronounced due to the lack of active check valve. Another class of micro-pumps, which does not rely on any moving parts, employ the interaction of fluid with an external field to induce continuous flow. The external field may be electrical (Santiago 2001; Chen and Santiago 2002) magnetic (Özbey et al. 2015; Doganay et al. 2020; Lemoff and Lee 2000), acoustic (Choe and Kim 2013; Wu et al. 2019) or chemical (Suzuki and Yoneyama 2003). However, the flexibility of continuous pumps is limited. For example, for electrokinetic pumping, the maximum electric field employed depends on the electrical conductivity of the liquid. This is to avoid the adverse effects of Joule heating, which may not compatible with a number of biological applications (Cetin and Li 2008). For magnetic actuation, the liquid needs to have specific magnetic properties and/ or contain magnetic particles (Doganay et al. 2020). For acoustic actuation, piezoelectric actuators need to be integrated within the microfluidic device and require relatively complex electronic hardware (Cetin et al. 2014; Choe and Kim 2013).

Despite the diversity of micro-pumps and actuation techniques available, syringe pumps along with pressure pumps have established themselves as the most viable option for microfluidic applications due to their simplicity and robustness (Laser and Santiago 2004; Iverson and Garimella 2008). Pressure pumps operate based on the modulation of air pressure in a sample reservoir. Even though pressure pumps provide a stable fluid flow, which may be quite important for some applications such as droplet generation
(Tan et al. 2006), they require expensive connectors, tubing as well as sensors and controllers, which comes at a cost. In addition, an infrastructure for pressurized air supply is also required for their operation. Considering the entire system with all the necessary components, a pressure pump may cost up to $\$ 10,000$ (Masterflex high-precision 2020). Recently, Thurgood et al. $(2018,2019)$ presented a pressure pump using latex balloons attached to syringes which reduce the cost of the system dramatically and makes the pressure pump suitable for low-resource settings. However, despite the simple instrumentation, the controlled flow rate requires the monitoring of the pressure inside the ballon, and the balloons occupy a bulky volume compared to the microfluidic device, especially when several balloons are used.

Syringe pumps rely on the displacement of a piston to deliver the sample from a disposable syringe to a microfluidic device. Commercial syringe pumps are typically delivered with integrated control electronics. They represent a relatively inexpensive solution with an entry price about $\$ 750 \mathrm{Ne}-1000$ programmable single syringe pump (2020). Since the liquid is incompressible, the liquid flow is solely controlled by the displacement of the piston. However, syringe pumps may also induce pulsation, especially at low flow rates, due to the friction between the cylinder of the syringe and the piston. Additionally, flow rate fluctuations may also be experienced due to the oscillations of the motor (depending on the quality of the hardware). However most of the time, these variations are tolerable and do not affect the performance of the operation. Another drawback of syringe pumps is that, especially in applications that require infusion of a cell suspension, cells sediment at the bottom of the syringe barrel during the operation, which hinders homogeneous dispensing. Magnetic stirrer integrated syringe pumps are available Syringe stirrer nemix (2020) or vertical syringe pumps may be used to address this issue.

In many microfluidic applications, rare and/or valuable samples, chemicals and reagents need to be introduced into the microfluidic device. In these situations, delivery of minute amount of valuable samples with minimum dead volume is vital. Although low-cost macro-scale syringes and tubings are easy and convenient to use with syringe and pressure pumps for microfluidic applications, they may create great amount of dead volume. Alternatively, micro-scale capillaries and syringes can be used; however, these components may be too expensive to be used as a disposable set; in addition, integration and sealing may require some special care (Uprocess syringe pump 2020). As an alternative to syringe and pressure pumps, pipetting is a very convenient and versatile option to precisely control the amount of liquid dispensed with zero dead volume during the operation. However, regular pipettors do not allow control of the flow rate. Recently, Kim et al. (2017) proposed and described the operation of a pipetting driven microfluidic 
immunohistochemistry platform. Throughout the process, a pipette was connected to the microfluidic platform with a PDMS adaptor, and the valuable sample in small size was infused into the microchannel. Kim and Choi (2016) introduced a "smart pipette" composed of a syringe (which was employed as the actuator), an air chamber and a conventional pipette tip (which was employed as the sample holding container). The volumetric flow rate was adjusted by the variable volume of the syringe; however, over the operation of $200 \mathrm{~s}$, the flow rate was observed to vary by $15 \%$. Therefore, if the precision on the flow rate does not affect the performance of the device, smart pipette is an excellent solution especially for low resource settings, but the accuracy of control of the flow rate is well below than that of syringe and pressure pumps.

Recently, Hatipoğlu et al. (2018) and Çetin et al. (2019) offered a low-cost, disposable sample loading interface named flexible hydraulic reservoir (FHR) which can be an attachment to any syringe available. FHR contains two types of fluids; the dummy fluid and the sample fluid, respectively. Dummy fluid is loaded both into the syringe and the interconnect tubing from the syringe to the FHR. The valuable fluid is loaded on the second compartment, which is isolated from the dummy fluid through an elastic membrane. FHR is connected to a microfluidic device to enable the infusion of all of the valuable liquid, which is deposited on the second compartment, into the microfluidic platform with zero-dead volume. In this previous version of FHR, the valuable liquid must be loaded to avoid air trapped between the membrane and the liquid, such that the valuable liquid can be delivered at a rate equal to that provided via the syringe. However, this requires a rigorous loading process, which is considered as a major drawback of FHR. One other drawback of this system was non-standard means of connection needed to interface the FHR with the microfluidic chip. FHR must be mounted directly onto the inlet of the microfluidic device to ensure zero-dead-volume dispensing of the valuable liquid to the device. These drawbacks seriously reduced the loading and dispensing versatility of the device. Here we address these issues by integrating a pipette tip to the FHRv2, thus enabling a world-to-chip interconnect for versatile sample loading and dispensing. Doing so, we combine flow rate control offered by the syringe pump, the zero dead-volume of FHR, and the versatility of pipetting.

The operating principle of FHRv2 is given in Fig. 1. As seen from the figure, the user does not need to load the sample into the syringe and interconnect tubing. Instead, they are always filled with a dummy fluid, and the user loads the sample into a pipette tip as with a regular pipette. The pipette tip is the only disposable component of the FHRv2. Integration of pipette tip introduces an important aspect of FHRv2, that is compartmentalization of the elastic membrane and the loaded liquid sample through

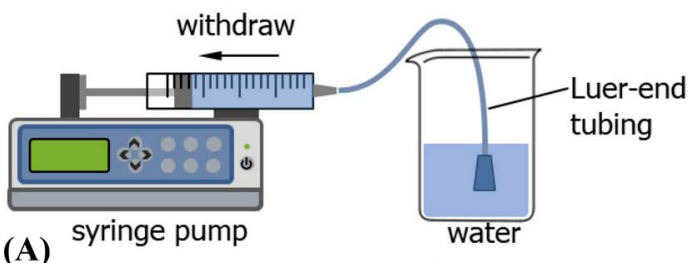

(A)
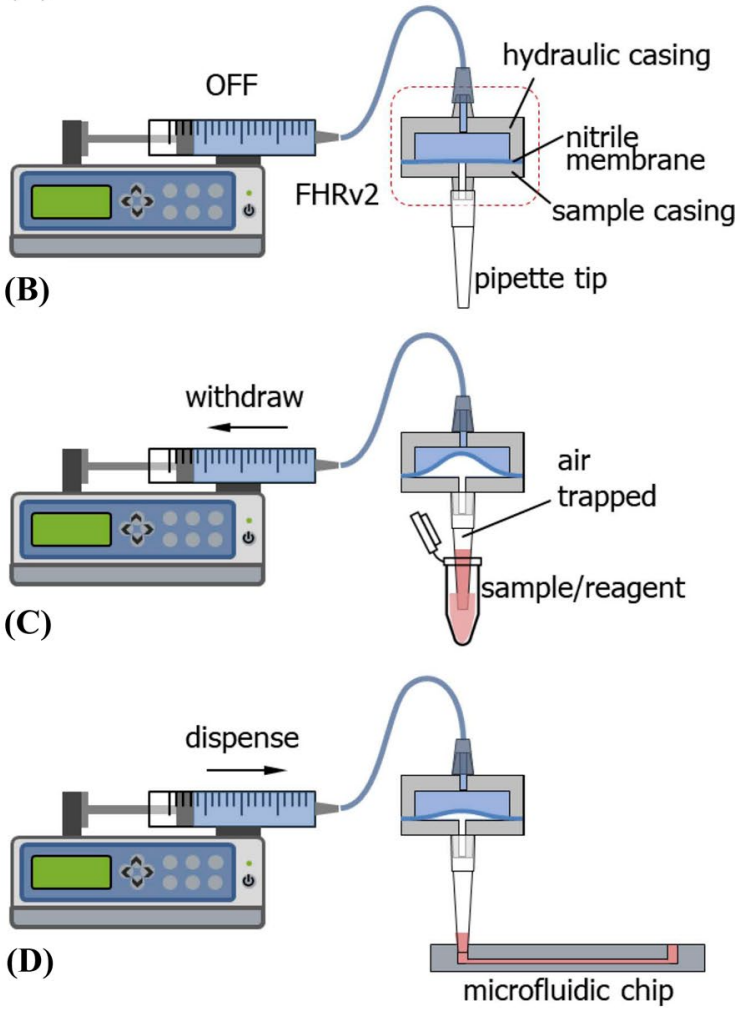

Fig. 1 Operating principles of FHRv2

air trapped in the pipette tip. This results in a capacitive behavior that damps out the potential oscillations due to the syringe pump. However, it also induces a time lag between the response of the syringe pump and the FHRv2. To predict the transient behavior of FHRv2 due to capacitive effect of air entrapped in the pipette tip (and the sample casing) during loading of the sample, a mathematical model relating the time-dependent flow rate at the outlet of the FHRv2 to the trapped air volume (which is equal to the internal volume of the pipette tip) and the infusion rate of the syringe pump. Through the mathematical model, developed in MATLAB 2019b Simulink environment, we observed that this lag time is in the order of $10 \mathrm{~s}$ with conventional pipette tips. However, both syringe pumps and FHRv2 reach steady state flow rate at approximately the same time due to a shorter settling time for the FHRv2, as explained below. Besides, the lag time of the FHRv2 can be minimized through the adjustment of the air volume (e.g. using a syringe needle instead of a pipette tip). The integration of the FHRv2 to a microfluidic device through 


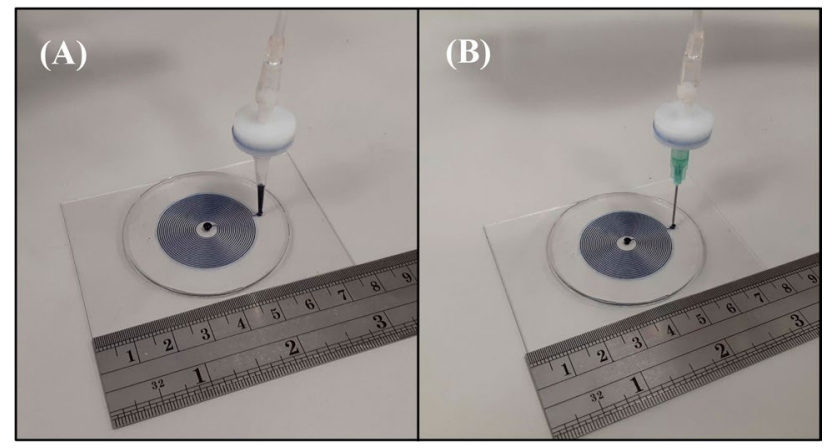

Fig. 2 The integration of the FHRv2 to a microfluidic device through $\mathbf{a}$ a pipette tip and $\mathbf{b}$ a syringe needle

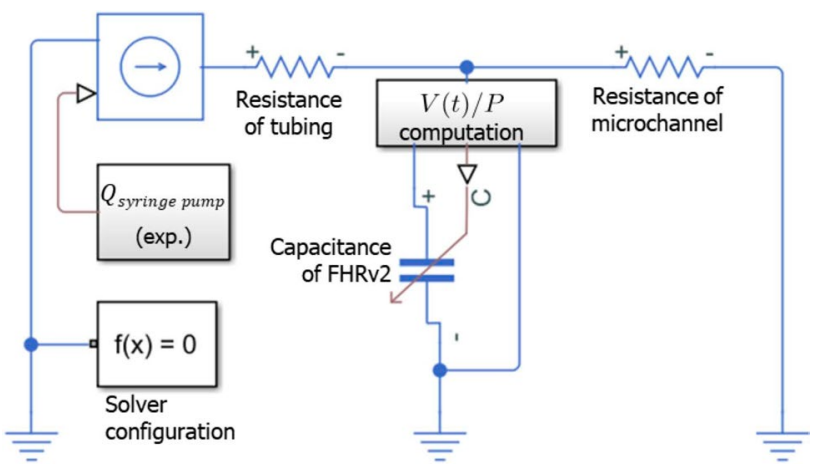

Fig. 3 MATLAB Simulink model used for predict the transient response of FHRv2. See ESI for the details of the model. $\dagger$

a pipette tip and a syringe needle is illustrated in Fig. 2. The model compares favorably with experiments during which the displaced volume is obtained by tracking the motion of a fluorescent dye-oil interface with an in-house developed image processing algorithm. We also developed a fabrication scheme as a first step in scalable manufacturing of FHRv2 by injection molding using polymer rapid tooling with a 3D printed mold. Finally, we demonstrated the use of FHRv2 to feature its feasibility in particle-based applications.

\section{Materials and methods}

\subsection{Mathematical model}

The mathematical model was built based on hydrodynamic-electrical circuit analogy using MATLAB R2019b Simulink (Fig. 3). The syringe pump was modeled as a current source, the tubing connecting the syringe to the
FHRv2 as a resistor, FHRv2 with the pipette tip as a capacitor, and the microchannel as a resistor, accordingly. The resistance of the tubing $\left(R_{\mathrm{t}}\right)$ was calculated as

$R_{\mathrm{t}}=\frac{128 \mu L_{\mathrm{t}}}{\pi D_{\mathrm{t}}^{4}}$

where $\mu$ is the dynamic viscosity of water, $L_{\mathrm{t}}$ and $D_{\mathrm{t}}$ are the length and the diameter of the tubing, respectively. Similarly, the resistance of a typically rectangular microchannel can be obtained using

$R_{\mathrm{c}}=\frac{12 \mu L_{\mathrm{c}}}{w h^{3}\left(1-\frac{0.63 h}{w}\right)}$,

where $L_{\mathrm{c}}$ is the wetted length of the microchannel, $w$ and $h$ are the width and the height of the microchannel, respectively. On the other hand, since we have utilized sufficiently long 0.7-mm ID tubing instead of a microchannel in flow characterization experiments (Fig. 5a), we used (Eq. (1)) to calculate the hydrodynamic resistance at the downstream of the FHRv2. The capacitance due to compliance of the tubing and the microchannel were neglected since the major capacitive effect is due to the air trapped shown in Fig. 1c. Moreover, the capacitive effect of the deformation of the nitrile membrane was ignored as the change in the volume underneath the membrane due to the deformation of the membrane was quite insignificant in comparison to its deflection Hatipoğlu et al. (2018). The capacitance of air trapped $\left(C_{\text {air }}\right)$ in the hydraulic circuit is

$C_{\text {air }}=\frac{V(t)}{P}$,

where $V(t)$ is the instantaneous volume and $P$ is the absolute pressure of the air trapped, respectively. To calculate the capacitance of air trapped in FHRv2, (Eq. (3)), the instantaneous volume and the absolute pressure need to be determined. To obtain the instantaneous volume of air trapped $(V(t))$, the integral of the instantaneous current (equivalent to the instantaneous flow rate, $Q(t)$ ) passing through the capacitor was subtracted from the initial volume of the air $\left(V_{\mathrm{air}, i}\right)$, since the current basically indicates the rate of the volume of air compressed. Similarly, to obtain the absolute pressure of the air trapped, voltage drop (equivalent to the pressure drop, $\Delta P$ ) across the capacitor was added to the atmospheric pressure $\left(P_{\text {atm }}\right)$. As a result, the capacitance of the air trapped was determined as

$C_{\text {air }}=\frac{V_{\mathrm{air}, i}-\int_{0}^{t} Q(t) \mathrm{d} t}{\Delta P+P_{\mathrm{atm}}}$.

This computation scheme introduces an algebraic loop in the model, which must be iteratively solved at each time step of 
the simulation. To ensure the convergence of the solution, initial value of the capacitor was set as

$$
C_{\text {air }, i}=\frac{V_{\text {air }, i}}{P_{\text {atm }}}
$$

\subsection{Fabrication via mechanical machining}

The hydraulic and sample casings of FHRv2 were fabricated by, respectively, turning and drilling a cylindrical polyimide stock of $25 \mathrm{~mm}$ diameter using a manual lathe (Opti D320 $\times 920$ lathe, Optimum Machinen, Germany). Inlet of the hydraulic casing and outlet of the sample casing were tapered in accordance with the dimensions of standard male Luer-cone connector and the neck of $250 \mu \mathrm{L}$ pipette tip. The dimensions of the hydraulic casing were set such that the inner volume was $120 \mu \mathrm{L}$. After machining, an O-ring was placed in the hydraulic casing as an elastic seat for to prevent leakage. Then the nitrile membrane was slightly stretched flat and bonded to the hydraulic casing by applying epoxybased adhesive. The sample casing was then adhesively bonded to the nitrile membrane to finalize the assembly. Figure 4a shows the exploded view and the cross section view



Fig. 4 a Exploded view, cross-sectional view of 3D CAD model and photo of assembly of mechanically machined FHRv2, b exploded view and cross-section view of 3D CAD model and photo of assembly of injection molded FHRv2, and c details of the 3D printed mold for injection molding of hydraulic casing and sample casing of FHRv2. See ESI for the STL files for both mechanically machined and injection molded FHRv2. $\dagger$ of the CAD model, and the assembly of FHRv2 fabricated by mechanical machining.

\subsection{Fabrication via plastic injection with 3D-printed mold}

The polymer tools for molding the hydraulic casing and the sample casing were printed using polyjet printer (StrataSYS J750, Stratasys Ltd) due to its high-feature size resolution and robustness. We used Vero ${ }^{\mathrm{TM}}$ range materials of the printer with gloss finish on the surface, as its low friction coefficient is ideal for part release. Post processing of the tools was undertaken using a jet washer (Genie Pro-600, Gemini Cleaning Systems Ltd) followed by air drying. To define the inner volume and the inlet hole of the hydraulic casing, a 3D printed core has been implemented. In addition, to define the outlet hole of the sample casing, a polymer pin was used as a core. Figure $4 \mathrm{c}$ shows the details of the 3D-printed molds and the cores. Once assembled, the mold with the core was fitted to the bolster plate on the injection molding machine (Microsystem 50, Battenfeld). Polypropylene (C711 70RNA, Braskem) was injected into the mold under 200 bar of holding pressure and $190{ }^{\circ} \mathrm{C}$ nozzle temperature to mold the hydraulic and sample casings.

Following the injection molding, the hydraulic and sample casings with nitrile membrane in between are brought together using

finger pressure until the parts are mechanically locked for assembly. By taking advantage of injection molding and 3D printing, we have revised the design of the FHRv2 such as to make the inner cavity of the hydraulic casing in a dome shape structure, to accommodate the nitrile membrane deformation during intake of the sample (Fig. 2c). We also revised the assembly scheme such that the sample casing could be snap fit into the hydraulic casing, while sandwiching the nitrile membrane in between. Figure $4 \mathrm{~b}$ shows the exploded view and the cross section view of the CAD model, and injection molded FHRv2. A protrusion that presses on the nitrile membrane was placed at the bottom of the hydraulic casing to prevent leakage (Fig. $4 \mathrm{~b}$ ).

\subsection{Flow characterization}

Figure 5a illustrates the test setup. with mechanically machined FHRv2 used in the experiments. A sufficiently long spiral test channel was utilized to measure the displaced volume by FHRv2 as a function of time. The test channel was formed by inserting an ID $0.7 \mathrm{~mm}$ tubing (the value of the diameter was also verified by microscope measurements) into a spiral shaped groove on a 3D printed template. The test channel was filled with oil prior to testing. Then, $60 \mu \mathrm{L}$ fluorescent dye was drawn into the pipette tip mounted at the outlet of the sample casing of the FHRv2 and the pipette 




Fig. 5 a Test setup used for flow characterization of FHRv2, and b illustration of the image processing scheme used to measure the volume dispensed

tip was fitted into the inlet of the test channel, such as to form a fluorescent dye-oil interface. During the test, the dye was injected through the test channel via FHRv2 by running the syringe pump (NE1002X, New Era Pump Systems) in dispense mode. The location of the fluorescent dye-oil interface, which can be related to the volume dispensed, was monitored by a camera (Phantom MIRO LC-110, Adept Turnkey Pty Ltd) at a rate of 30 fps under UV-A illumination to enhance the contrast between the dye and oil. Captured frames were then analyzed using a custom image processing script written on MATLAB to determine the location of the interface with respect to time. The same experiment was carried out in the absence of FHR, such that the test channel was directly connected to the tip of the syringe, to measure the volume delivered by the syringe pump. Due to uncertainties in locating the interface by image processing and possible non-uniformities of the internal surface of the tubing, fluctuations were observed in the measured volume at $30 \mathrm{fps}$ capturing frequency. The data were decimated by 20 to eliminate the noise, while preserving the trend.

Total volume dispensed was determined by processing the data obtained from the camera. The image processing was performed to measure the distance covered by the interphase between fluorescent dye and oil in the test channel at each frame, and then summing up the incremental distances over frames. In this process, first the frames were converted to binary images. Then the coordinates of the boundary of the interphase were determined by means of built-in MATLAB function bwboundaries. Once the coordinates of the interphase was determined, the angular $(\theta)$ and the radial $(R)$ coordinates of each pixel in the vicinity of the interphase with respect to the center of the spiral (as the center of the coordinate frame) were computed to generate a plot of $\theta$ vs. $r$, as shown Fig. 5b. Based on these data, a mean angle $\bar{\theta}$ and a mean radius $\bar{r}=\left(r_{\min }+r_{\max }\right) / 2$ were computed. At each frame, the pixel with the minimum $\theta$ was found and the neighbor of this point (indicated by yellow circles in Fig. 5b) which has a $\theta$ coordinate within a defined tolerance is included in the calculation of the mean angle as $\theta_{\text {interphase }}$. The uncertainty of $\theta$ and $r$ coordinates of each point is determined by assigning \pm lpixel in the normal and tangential directions. Having computed $\bar{\theta}$ and $\bar{r}$ and the associated uncertainty values, the differential distance covered by the interface in each frame was computed, which was related to instantaneous change of the volume dispensed. With the assumption of constant cross-sectional area of the tubing, sum of instantaneous change of the volume yielded volume dispensed up to frame $j,\left(V_{j}\right)$ :

$V_{j}=\sum_{i=1}^{j}\left(\bar{\theta}_{i}-\bar{\theta}_{i-1}\right) \bar{r}_{i}(\pi / 4) D_{\mathrm{t}}^{2}$.

The uncertainty in the volume dispensed $\left(\Delta V_{j}\right)$ was calculated by using the uncertainty associated with the measurement of the mean angular and radial positions, $\bar{\theta}_{i}, \bar{\theta}_{i-1}$ and $\bar{r}_{i}$ as

$$
\Delta V_{j}=\sqrt{\sum_{i=1}^{j}\left[\left(\frac{\partial V_{j}}{\partial \bar{\theta}_{i}} \Delta \bar{\theta}_{i}\right)^{2}+\left(\frac{\partial V_{j}}{\partial \bar{\theta}_{i-1}} \Delta \bar{\theta}_{i-1}\right)^{2}+\left(\frac{\partial V_{j}}{\partial \bar{r}_{i}} \Delta \bar{r}_{i}\right)^{2}\right]} .
$$

\subsection{Particle dispensing experiments}

A custom PDMS microfluidic chip was fabricated for particle dispensing experiments as shown in Fig. 6. Total volume of the chip was approximately $20 \mu \mathrm{L}$ with $50 \mu \mathrm{m}$ channel height. $13 \mu \mathrm{m}$ fluorescent particles with known concentrations were dispensed into the chip using a syringe pump with and without FHRv2. To measure the concentration of the loaded particles, $25 \mu \mathrm{L}$ samples were taken from the prepared suspension loaded onto a hemocytometer and the particles were counted. To measure the concentration of the particles dispensed into the chip, a known region of interest on the chip was recorded at $30 \mathrm{fps}$ during the flow. Captured frames (total of 800 frames per each experiment) were decimated by 20 , resulting in 40 frames to be processed. Builtin MATLAB function bwboundaries was utilized to locate the particles in the frames (see Fig. 6b, c). Identified 


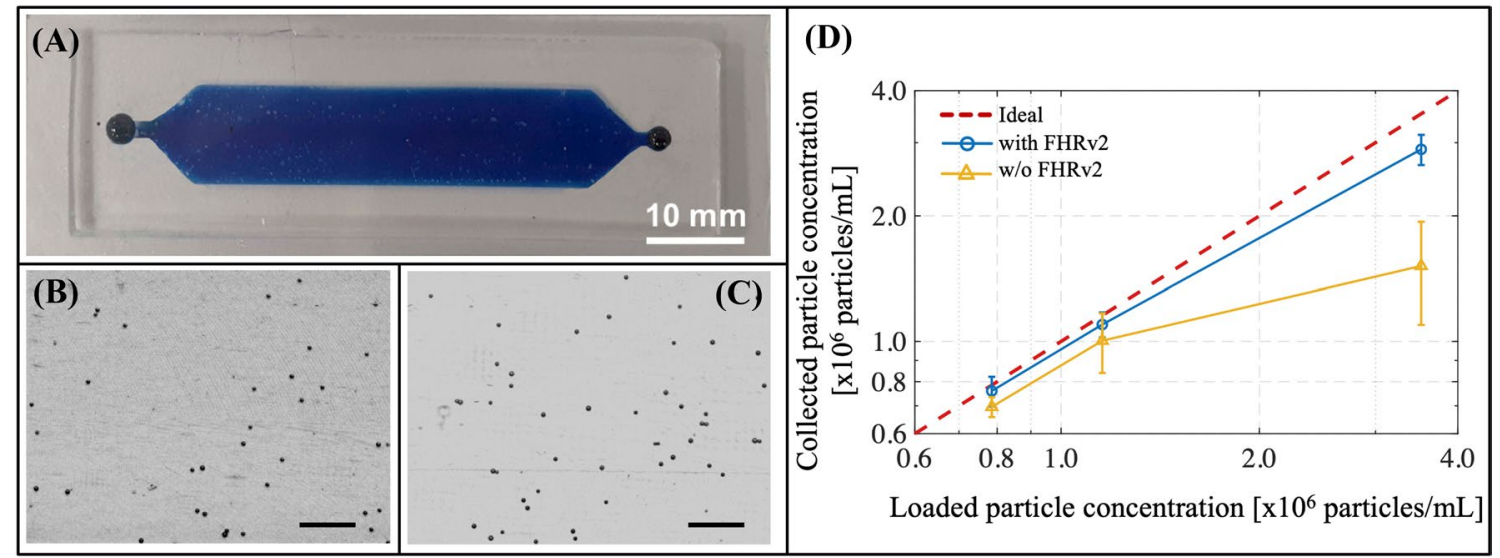

Fig. 6 b Test chip used for particle dispensing experiments. Sample frames captured during particle dispensing b without FHRv2 and with c FHRv2. Loaded particle concentration is $0.76 \times 10^{6}$, scale bar

particles were counted and the particle count was divided by the volume of liquid in the region of interest to calculate the particle concentration in each processed frame. Average of the processed frames was identified as the collected particle concentration as shown in Fig. 6d. The standard deviation of the concentration among the processed frames was used to define the error bars in Fig. 6d.

\section{Results and discussion}

To characterize the flow response of FHRv2, we measured the volume dispensed by FHRv2 at different volumetric flow rates and compared the results with a bare syringe pump. The results for three different volumetric flow rate values $(Q=20,40,60 \mu \mathrm{L} / \mathrm{min})$ are displayed in Fig. 7. As seen from the figure, the syringe pump and FHRv2 curves becomes linear and parallel to each other after a transient period. FHRv2 has a longer transient response for all three cases, due to the capacitive behavior induced by the trapped air. The results of MATLAB Simulink model for the FHRv2 with $250 \mu \mathrm{L}$ pipette tip are also presented in the figure. To compare the experimental results and the mathematical model, time derivative of the volume delivered by the syringe pump was input to Simulink model. With this approach, an excellent agreement is observed between the experimental data and the model. Therefore, once a user characterizes the syringe pump at hand, the performance of the FHRv2 can be predicted for a given set of operating parameters.

To demonstrate the effect of the air trapped on the performance of FHRv2, two cases; $1.5^{\prime \prime}$ long $18 \mathrm{G}$ blunt needle with ID $0.84 \mathrm{~mm}$ (corresponding to $45 \mu \mathrm{L}$ inner volume including the Luer hub) and $250 \mu \mathrm{L}$ pipette tip were simulated. The experimental flow rate together with the simulated flow rate are given as a function of time in Fig. 8. indicates $200 \mu \mathrm{m}$. d Collected particle concentration versus loaded particle concentration at $Q=40 \mu \mathrm{L} / \mathrm{min}$. Dash line indicates the reference
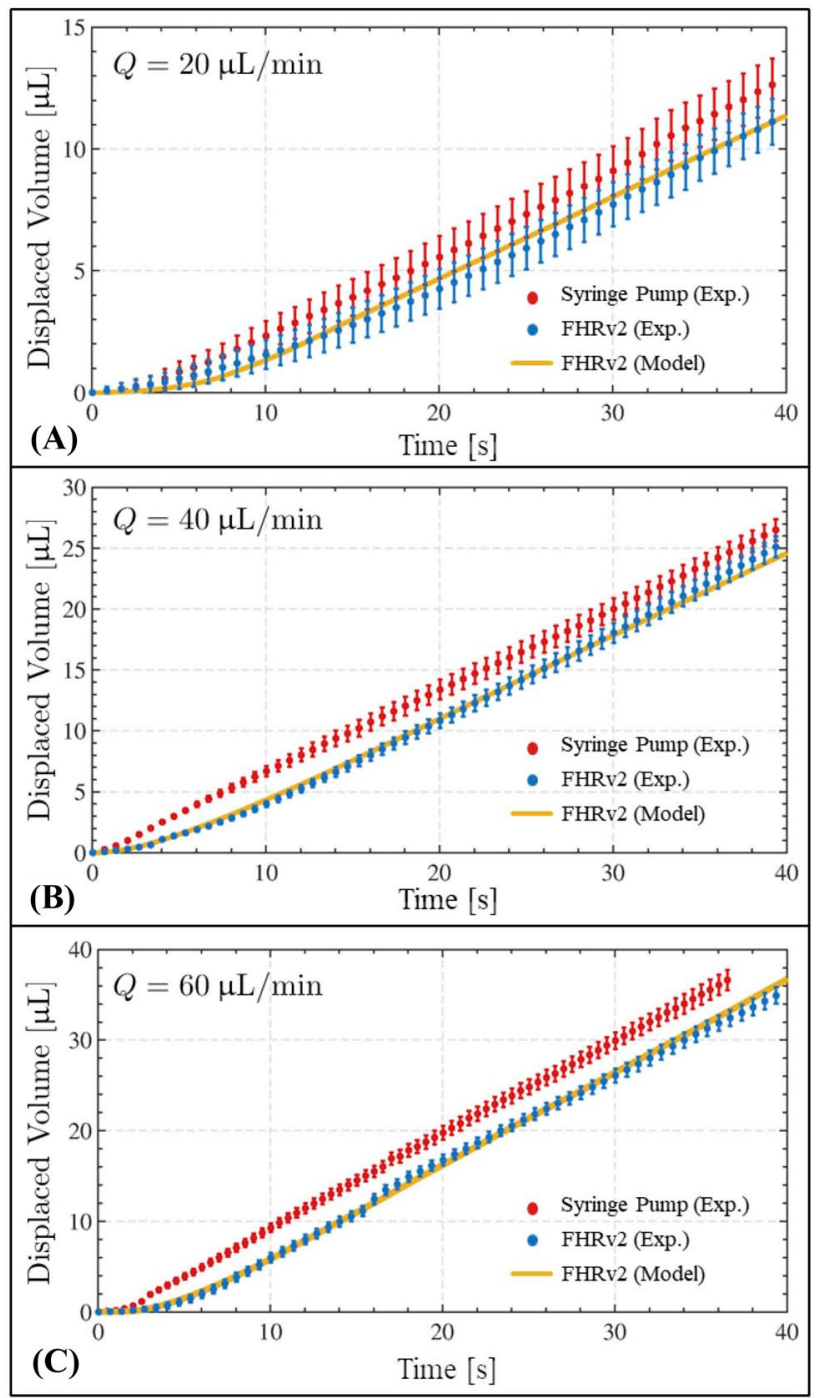

Fig. 7 Displaced volume as a function of time: a $Q=20 \mu \mathrm{L} / \mathrm{min}$, b $Q=40 \mu \mathrm{L} / \mathrm{min}, \mathbf{c} Q=60 \mu \mathrm{L} / \mathrm{min}$ 



Fig. 8 Simulated flow rate as a function of time: $\mathbf{a} Q=20 \mu \mathrm{L} / \mathrm{min}, \mathbf{b}$ $Q=40 \mu \mathrm{L} / \mathrm{min}, \mathbf{c} Q=60 \mu \mathrm{L} / \mathrm{min}$

$10 \%$ envelope around the steady-state flow rate value is also shown on the figure (dotted line). All the curves show similar time-dependent behavior, with a rise time $\left(t_{\mathrm{r}}\right)$ followed by a settling time (the instant when the response fall within the envelope) before reaching the target flow rate within the envelope. However, due to the capacitive behavior of the air trapped, several observations can be deduced:

- The rise time for the FHRv2 is longer than that of the syringe pump, and the rise time increases with increasing air volume.

- The settling time is longer for the syringe pump, and it decreases with increasing air volume.

- The oscillations damp out with the increased air volume both in the transient regime and at steady-state. Especially for the case with larger air volume, the flow rate reaches the steady-state value with a relatively shorter settling period.

Although rise time increases with the introduction of the FHRv2, the settling period decreases compared to the syringe pump. Therefore, the overall transient time for the syringe pump and FHRv2 is similar ( $<20 \mathrm{~s}$ for all cases). This result confirms that the capacitive behavior of FHRv2 will not negatively impact microfluidic applications that currently rely on syringe pumps. Additionally, the oscillations observed in the data are due to the syringe pump and are expected to improve with better pumps, which would result in even smoother FHRv2 response.

In addition to flow characterization, we demonstrated the functionality of FHRv2 to prevent non-homogeneous particle dispensing due to sedimentation in typical syringe pumps. For this purpose, we dispensed fluorescent particles at $Q=40 \mu \mathrm{L} / \mathrm{min}$ flow rate into a custom test chip using a syringe pump (Fig. 6a) and a FHRv2 connected to a syringe pump. Figure $6 \mathrm{~d}$ reveals that concentration of collected particles is considerably smaller than the loaded concentration when using the syringe pump indicating that significant amount of particles sediment at the syringe barrel. A reduction of $\sim 50 \%$ is observed for a particle concentration of $\sim 3.5 \times 10^{6}$ particles $/ \mathrm{mL}$. In comparison, the concentration of the collected particles is much closer to the loaded particles concentration when using the FHRv2. FHRv2 is advantageous in particle dispensing applications as sedimentation problem is intrinsically eliminated due to default vertical orientation of FHRv2. However, we observed a considerable particle loss $(\sim 10 \%)$ in relatively higher concentrations of particles $\left(\sim 3.5 \times 10^{6}\right.$ particles $\left./ \mathrm{mL}\right)$ (Fig. $\left.6 \mathrm{~d}\right)$. Noting that the particles were only contained in the pipette tip and did not interact with FHRv2, we concluded that the loss cannot be related to the geometry or construction of FHRv2. Therefore, this loss is mainly attributed to the errors in experimental procedure.

FHRv2 characterized in flow experiments (Fig. 7) were fabricated by mechanical machining (Fig. 4a), which is typically preferred as an early-stage prototyping method Lee et al. (2018). However, some applications including any dispensing of clinical samples may require a single-use FHRv2. In such cases, a scalable manufacturing method should be adopted to allow higher fabrication volume. For this purpose, we have developed an injection molding-based manufacturing scheme that utilized 3D-printed polymer tools. This approach represents an intermediate step to a scalable alternative.

Injection molding is commonly accepted as the "gold standard" for the fabrication of high volume, low unit cost polymer devices Lee et al. (2018). On the other hand, high cost of steel tooling is not justified in prototyping or low to medium volume production. Polymer additive rapid tooling 
that reduces the costs in injection molding was first proposed nearly 20 years ago Harris et al. (2002), but it only received considerable interest in recent years due to the democratization of 3D printing Rahmati (2014); Anurag et al. (2019). However, very little has been done for geometrically complex, precision micro-devices. Here, we demonstrate the use of 3D-printed tools in injection molding to manufacture FHRv2 (Fig. 4b, c). We show that despite the low cost of the polymer tool, we were able to maintain feature quality as illustrated in Fig. 4b. We tested injection molded FHRv2 against leakage by applying pressure at the inlet of the hydraulic casing and showed that injection-molded FHRv2 could withstand a pressure of 0.4 bars without leakage.

\section{Conclusions}

A second-generation FHR that combines continuous pumping, zero-dead volume and the versatility of pipetting is developed. The performance of FHRv2 is demonstrated in comparison with a syringe pump at flow rates ranging between 20 and $60 \mu \mathrm{L} / \mathrm{min}$. FHRv2 provides smoother operation and identical transient time to reach steady flow rate as confirmed by the mathematical model. In addition, FHRv2 prevents sedimentation-induced artifacts typically encountered in typical syringe pumps when dispensing particles. The fabrication of the FHRv2 with injection molding using a 3D-printed mold is also demonstrated. Considering all the aspects, FHRv2 is very user-friendly and has a flexible design. It can be implemented with any syringe pump, which is available in almost every microfluidics research environment. It can also be introduced as a very inexpensive set (especially fabricated by injection molding) in a commercial microfluidic product to serve as an interface between the fluid pumping equipment and microfluidic chip. Additionally, since FHRv2 is inherently mounted on a microfluidic chip in vertical orientation, it enables homogeneous delivery of suspension in case of particle or cell based applications. The design of FHRv2 is quite flexible and can easily be modified, and through the mathematical model, the response of FHRv2 can be predicted for a specific application. Our FHRv2 opens up new opportunities in low-cost sample handling in microfluidic devices. Its zero dead volume and smooth operation make it particularly suited for handling small amount of precious fluids.

Acknowledgements This study is financially supported by the Turkish Scientific and Technical Research Council, under Grant no. 118E023. B.Ç. would like to acknowledge fundings from the Turkish Academy of Sciences through Outstanding Young Scientist Program (TÜBAGEBIP) and The Science Academy, Turkey, through Distinguished Young Scientist Award (BAGEP). JC and KC would like to acknowledge funding from the Newton Fund-Institutional Links grant (ID 352360246) for the experimental work on the fabrication of the FHRv2 with rapid prototyping injection moulding. The authors would like to thank Dr. Vannessa Goodship for her support in injection molding.

Author contributions All authors contributed the writing and editing of the manuscript. BÇ and EY conceived the idea and developed the mathematical model. AA, AT and BS designed the experimental setup and conducted the experiments. BÇ and AA performed image processing. Mechanical machining of the FHRv2 was performed by EY. 3D printing of the mold and injection molding of the FHRv2 was carried out by $\mathrm{JC}$ and $\mathrm{KC}$.

\section{Compliance with ethical standards}

Conflict of interest There are no conflicts to declare.

\section{References}

Aggarwal S, Paul BE, DasGupta A, Chatterjee D (2016) Experimental characterization of piezoelectrically actuated micromachined silicon valveless micropump. Microfluid Nanofluid 21:2

Albrecht DR, Bargmann CI (2011) High-content behavioral analysis of Caenorhabditis elegans in precise spatiotemporal chemical environments. Nat Methods 8:599-605

Amrani I, Cheriet A, Feliachi M (2018) Design and experimental investigation of a bi-directional valveless electromagnetic micro-pump. Sens Actuator A Phys 272:310-317

Anurag B, Dirk P, Don C, Digby S (2019) A methodology for setting the injection moulding process parameters for polymer rapid tooling inserts. Rapid Prototyp J 25:1493-1505

Baraket A et al (2013) Development of a flexible microfluidic system based on a simple and reproducible sealing process between polymers and poly(dimethylsiloxane). Microelectron Eng 111:332-338

Bhatia SN, Ingber DE (2014) Microfluidic organs-on-chips. Nat Biotechnol 32:760-772

Cetin B, Li D (2008) Effect of joule heating on electrokinetic transport. Electrophoresis 29:994-1005

Cetin B, Ozer MB, Solmaz ME (2014) Microfluidic bio-particle manipulation for biotechnology. Biochem Eng J 92:63-82

Çetin B, Yıldırım E, Hatipoğlu U (2019) İ.D. Bilkent University and Cankaya University, PCT/TR2019/050142, patent pending

Chen Chuan-Hua, Santiago J G (2002) A planar electroosmotic micropump. A planar electroosmotic micropump. J Microelectromech Syst 11:672-683

Chin CD, Linder V, Sia SK (2012) Commercialization of microfluidic point-of-care diagnostic devices. Lab Chip 12:2118-2134

Choe Y, Kim ES (2013) Valveless micropump driven by acoustic streaming. J Micromech Microeng 23:045005

Doganay S, Cetin L, Ezan MA, Turgut A (2020) A rotating permanent magnetic actuator for micropumping devices with magnetic nanofluids. J Micromech Microeng 30:075012

Gong MM, MacDonald BD, Vu Nguyen T, Sinton D (2012) Handpowered microfluidics: a membrane pump with a patient-to-chip syringe interface. Biomicrofluidics 6:04412

Guevara-Pantoja PA, Jiménez-Valdés RJ, Garcia-Cordero JL, Caballero-Robledo GA (2018) Pressure-actuated monolithic acrylic microfluidic valves and pumps. Lab Chip 18:662-669

Harris R, Hopkinson N, Newlyn H, Hague R, Dickens P (2002) Layer thickness and draft angle selection for stereolithography injection mould tooling. Int J Prod 40:719-729

Hatipoğlu U, Çetin B, Yıldırım E (2018) A novel zero-dead-volume sample loading interface for microfluidic devices: flexible 
hydraulic reservoir (FHR). J Micromech Microeng 28:097001. https://doi.org/10.1088/1361-6439/aac333

Herrlich S, Spieth S, Messner S, Zengerle RS (2012) Osmotic micropumps for drug delivery. Adv Drug Deliv Rev 64:1617-1627

Huh D et al (2013) Microfabrication of human organs-on-chips. Nat Protoc 8:2135-2157. https://doi.org/10.1038/nprot.2013.137

Iverson BD, Garimella SV (2008) Recent advances in microscale pumping technologies: a review and evaluation. Microfluid Nanofluid 5:145-174

Kim B, Choi S (2016) Smart pipette and microfluidic pipette tip for blood plasma separation. Small 12:190-197

Kim J-H, Na K-H, Kang C, Kim Y-S (2005) A disposable thermopneumatic-actuated micropump stacked with PDMS layers and ito-coated glass. Sens Actuator A Phys 120:365-369

Kim S, Kwon S, Cho CH, Park J-K (2017) Pipetting-driven microfluidic immunohistochemistry to facilitate enhanced immunoreaction and effective use of antibodies. Lab Chip 17:702-709. https://doi. org/10.1039/C6LC01495J

Laser DJ, Santiago JG (2004) A review of micropumps. J Micromech Microeng 14:R35-R64

Lee UN et al (2018) Fundamentals of rapid injection molding for microfluidic cell-based assays. Lab Chip 18:496-504

Lemoff AV, Lee AP (2000) An ac magnetohydrodynamic micropump. Sens Actuator B Chem 63:178-185

Ma T, Sun S, Li B, Chu J (2019) Piezoelectric peristaltic micropump integrated on a microfluidic chip. Sens Actuator A Phys 292:90-96

Masterflex high-precision, high-pressure piston pumps (2020). https ://www.coleparmer.com/p/masterflex-high-precision-high-press ure-piston-pumps $/ 66368 ? \mathrm{Ntt}=$ pressure+pump. Accessed 11 June 2020

Mi S, Pu H, Xia S, Sun W (2020) A minimized valveless electromagnetic micropump for microfluidic actuation on organ chips. Sens Actuator A Phys 301:111704

Moorthy J, Beebe D (2003) In situ fabricated porous filters for microsystems. Lab Chip 3:62-66

$\mathrm{Ne}-1000$ programmable single syringe pump (2020). http://www.syrin gepump.com/NE-1000.php. Accessed 11 June 2020

Ni J, Wang B, Chang S, Lin Q (2014) An integrated planar magnetic micropump. Microelectron Eng 117:35-40

Nisar A, Afzulpurkar N, Mahaisavariya B, Tuantranont A (2008) Mems-based micropumps in drug delivery and biomedical applications. Sens Actuator B Chem 130:917-942

Özbey A, Karimzadehkhouei M, Yalçın SE, Gözüaçık D, Koşar A (2015) Modeling of ferrofluid magnetic actuation with dynamic magnetic fields in small channels. Microfluid Nanofluid 18:447-460

Rahmati S (2014) 10.12-direct rapid tooling. In: Hashmi S, Batalha GF, Tyne CJV, Yilbas B (eds) Comprehensive materials processing. Elsevier, New York, pp 303-344
Rusli M, Chee PS, Arsat R, Lau KX, Leow PL (2018) Electromagnetic actuation dual-chamber bidirectional flow micropump. Sens Actuator A Phys 282:17-27

Santiago JG (2001) Electroosmotic flows in microchannels with finite inertial and pressure forces. Anal Chem 73:2353-2365

Sen M, Wajerski D, Gad-el Hak M (1996) A novel pump for MEMS applications. J Fluids Eng J Fluids Eng 118:624-627

Suzuki H, Yoneyama R (2003) Integrated microfluidic system with electrochemically actuated on-chip pumps and valves. Sens Actuator B Chem 96:38-45

Syringe stirrer nemix (2020). https://www.cetoni.com/products/syrin ge-stirrer-nemix/. Accessed 11 June 2020

Tan Y-C, Cristini V, Lee AP (2006) Monodispersed microfluidic droplet generation by shear focusing microfluidic device. Sens Actuator B Chem 114:350-356

Tang Z, Shao X, Huang J, Yao J, Ding G (2020) Manipulate microfluid with an integrated butterfly valve for micropump application. Sens Actuator A Phys 306:111965

Thurgood P et al (2018) A self-sufficient pressure pump using latex balloons for microfluidic applications. Lab Chip 18:2730-2740

Thurgood P et al (2019) Self-sufficient, low-cost microfluidic pumps utilising reinforced balloons. Lab Chip 19:2885-2896

Uprocess syringe pump sps01 (2020). https://products.labsmith.com/ uprocess-syringe-pump-sps01/\#.XuMuP0UzaUl. Accessed 12 June 2020

Weibel DB, Siegel AC, Lee A, George AH, Whitesides GM (2007) Pumping fluids in microfluidic systems using the elastic deformation of poly(dimethylsiloxane). Lab Chip 7:1832

Wu Z et al (2019) A digital acoustofluidic pump powered by localized fluid-substrate interactions. Anal Chem 91:7097-7103

Ye Y, Chen J, Ren YJ, Feng ZH (2018) Valve improvement for high flow rate piezoelectric pump with PDMS film valves. Sens Actuator A Phys 283:245-253

Zhou P, Young L, Chen Z (2010) Weak solvent based chip lamination and characterization of on-chip valve and pump. Biomed Microdevices 12:821-832

Zimmermann M, Schmid H, Hunziker P, Delamarche E (2007) Capillary pumps for autonomous capillary systems. Lab Chip 7:119-125

Publisher's Note Springer Nature remains neutral with regard to jurisdictional claims in published maps and institutional affiliations. 